\title{
Consistent Self-monitoring of Weight: A Key Component of Successful Weight Loss Maintenance
}

\author{
Meghan L. Butryn,* Suzanne Phelan, $\dagger$ James O. Hill, $\neq$ and Rena R. Wing $\dagger$
}

\begin{abstract}
\end{abstract}
Objective: The objectives were to investigate the characteristics associated with frequent self-weighing and the relationship between self-weighing and weight loss maintenance.

Research Methods and Procedures: Participants $(n=$ 3003) were members of the National Weight Control Registry (NWCR) who had lost $\geq 30 \mathrm{lbs}$, kept it off for $\geq 1$ year, and had been administered the self-weighing frequency assessment used for this study at baseline (i.e., entry to the NWCR). Of these, $82 \%$ also completed the one-year follow-up assessment.

Results: At baseline, $36.2 \%$ of participants reported weighing themselves at least once per day, and more frequent weighing was associated with lower BMI and higher scores on disinhibition and cognitive restraint, although both scores remained within normal ranges. Weight gain at 1-year follow-up was significantly greater for participants whose self-weighing frequency decreased between baseline and one year $(4.0 \pm 6.3 \mathrm{~kg})$ compared with those whose frequency increased $(1.1 \pm 6.5 \mathrm{~kg})$ or remained the same $(1.8 \pm 5.3 \mathrm{~kg})$. Participants who decreased their frequency of self-weighing were more likely to report increases in

*Department of Psychology, Drexel University, Philadelphia, Pennsylvania; $†$ Department of Psychiatry and Behavioral Medicine, Brown Medical School and Miriam Hospital, Providence, Rhode Island; and \$Center for Human Nutrition, University of Colorado at Denver, Denver, Colorado. their percentage of caloric intake from fat and in disinhibition, and decreases in cognitive restraint. However, change in self-weighing frequency was independently associated with weight change.

Discussion: Consistent self-weighing may help individuals maintain their successful weight loss by allowing them to catch weight gains before they escalate and make behavior changes to prevent additional weight gain. While change in self-weighing frequency is a marker for changes in other parameters of weight control, decreasing self-weighing frequency is also independently associated with greater weight gain.

Key words: weight regain, behavioral strategies

\section{Introduction}

Previous research has identified several behaviors associated with long-term weight loss maintenance. Much of this research has been conducted with the National Weight Control Registry (NWCR) ${ }^{1}$, a database of successful dieters created in 1994 by Wing and Hill (1). To be enrolled in the NWCR, individuals must have successfully lost at least 30 lbs and maintained that weight loss for at least 1 year. Several behaviors are characteristic of these successful weight losers. They typically report that they eat a low-fat and low-calorie diet (1). Approximately four fifths of participants report that they eat breakfast every day (2). NWCR participants are characterized by their high levels of physical activity; on average, they exercise $\sim 1$ hour per week, typically by engaging in walking (1). The majority of participants $(62 \%)$ report watching 10 or fewer hours of television per week, which is substantially less than average for an American adult (3).

An additional behavior that characterizes NWCR participants is regular self-monitoring of weight. One of the

${ }^{1}$ Nonstandard abbreviation: NWCR, National Weight Control Registry. 
earliest studies conducted in the NWCR $(n=784)$ found that $44 \%$ of participants weighed themselves at least once per day and $31 \%$ weighed themselves once per week (1). Most self-regulation theories consider self-monitoring an essential element of behavioral self-regulation (4,5). Weight monitoring may allow individuals to notice how specific situations or patterns of eating or physical activity behaviors relate to changes in body weight. Weight monitoring also provides an opportunity for positive reinforcement when changes in behavior correspond to weight loss or avoidance of weight gain.

Preliminary data suggest that frequent self-monitoring of body weight is associated with improved weight control. For participants enrolled in a weight gain prevention trial and obese participants enrolled in a weight loss trial, regular weight monitoring was associated with less weight gain and greater weight loss, respectively (6). Similarly, among participants in an intervention to prevent weight regain, those who weighed themselves daily had decreased risk of weight regain $(7)$.

The goal of the current study was to investigate more fully the characteristics of frequent self-weighers and the relationship between self-weighing and weight change in a larger sample of successful weight losers in the NWCR. Specifically, this study aimed to determine whether baseline self-weighing frequency or change in self-weighing frequency was related to weight loss maintenance over 1 year of follow-up.

\section{Research Methods and Procedures}

\section{Participants}

Participants in the NWCR were continuously recruited through media advertisements from 1993 to 2004 and were not compensated for completing assessments. To be included in the current study, participants must have been enrolled in the NWCR long enough to complete a 1-year follow-up and not have reported a pregnancy within the subsequent year. Of the 4051 participants who met these criteria, $3003(74 \%)$ were administered the self-weighing frequency assessment and, thus, were included in this study; 2462 of the $3003(82 \%)$ also provided data at 1 year. Independent sample $t$ tests indicated that participants who completed 1-year follow-up, compared with those who did not, had significantly higher age (48.7 \pm 12.4 vs. $44.5 \pm$ 12.7 years), lower BMI (25.1 \pm 4.8 vs. $\left.26.0 \pm 5.1 \mathrm{~kg} / \mathrm{m}^{2}\right)$, lower weight $(71.9 \pm 16.2$ vs. $74.9 \pm 17.5 \mathrm{~kg})$, smaller weight loss from maximum $(33.1 \pm 18.0$ vs. $35.4 \pm 18.8$ $\mathrm{kg})$, and longer weight maintenance duration (73.0 \pm 96.1 vs. $55.5 \pm 71.4$ months) (all $p$ values $<.05$ ). $\chi^{2}$ tests also indicated that completers were significantly more likely to be white than non-completers (95.4\% vs. 92.1\%); there were no significant differences between groups by gender.

\section{Assessments}

Participants completed self-report questionnaires at baseline (i.e., entry into the NWCR) and 1-year follow-up. Weight and height were self-reported. The reliability and validity of self-reported weight in NWCR participants has been reported previously; correlations between documented weight and self-reported weight were 0.98 for maximum weight and 0.97 for current weight (8). Self-weighing frequency was assessed at baseline and 1-year follow-up with the question, "Currently, how often do you weigh yourself?" Response options, which included several times/day, 1 time/day, several times/wk, 1 time/wk, $<1$ time/wk, and $<1$ time/mo, were collapsed into 3 categories for data analysis: at least daily, several times per week or weekly, and less than weekly.

A self-report questionnaire was administered to assess gender, ethnicity, age, education level, and weight history. The Three Factor Eating Questionnaire was administered to assess disinhibition and cognitive restraint (9). The internal consistency and test-retest reliability of these scales have been well documented $(9,10)$. Calories expended through physical activity were assessed with the Paffenbarger Activity Questionnaire, which has demonstrated adequate testretest reliability and is significantly correlated with tests of cardiovascular fitness (11-13). Dietary intake was assessed with the Block Food Frequency Questionnaire, which provides estimates of daily energy intake and percentage of energy intake from fat. This measure has adequate testretest reliability (14), and changes in this measure are correlated with estimates obtained from 4-day food diaries $(15,16)$.

\section{Statistics}

SPSS version 13.0 for Windows (SPSS, Inc., Chicago, IL) was used for all analyses. ANOVA and $\chi^{2}$ analyses were used to examine differences between groups at baseline. ANOVA was used to examine changes in weight from baseline to 1-year follow-up, with Tukey post hoc tests as appropriate. Where noted, covariates also were entered into these analyses.

\section{Results}

\section{Self-Weighing at Entry to the NWCR}

Characteristics of participants in this sample are shown in Table 1. At entry to the NWCR, participants had been maintaining their weight loss, on average, for $5.8 \pm 7.7$ years. At baseline, $36.2 \%$ of participants reported weighing themselves at least once per day, $42.5 \%$ reported weighing themselves less than daily but at least weekly, and $21.3 \%$ reported weighing themselves less than once per week. Differences at baseline between participants who weighed themselves at least daily, several times per week or weekly, and less than weekly were examined and are shown in Table 
Table 1. Characteristics of participants at baseline

\begin{tabular}{lccc}
\hline \multicolumn{1}{c}{ Characteristic } & \% & Mean & SD \\
\hline Gender (\% female) & 75.1 & - & - \\
Ethnicity (\% white) & 94.8 & - & - \\
Education (\% completed) & & - & - \\
$\quad$ High school & 10.7 & - & - \\
$\quad$ Some college & 24.8 & - & - \\
$\quad$ College & 26.2 & - & - \\
$\quad$ Graduate or professional & 31.5 & - & - \\
Age (yrs) & - & 48.0 & 12.6 \\
Maximum BMI & - & 37.1 & 8.8 \\
BMI at baseline & - & 25.3 & 4.8 \\
Duration of weight loss & & & \\
$\quad$ maintenance $(y r s)$ & - & 5.8 & 7.7
\end{tabular}

$\mathrm{SD}$, standard deviation.

2. More frequent weighing was associated with older age, lower BMI at baseline, lower BMI at maximum lifetime weight, shorter duration of weight loss maintenance, greater cognitive restraint, greater disinhibition, and greater fat intake. Additionally, participants who weighed themselves at least daily, several times per week or weekly, and less than weekly differed by gender $(70.8 \%, 75.6 \%$, and $81.5 \%$ female, respectively, $p<0.001)$ and ethnicity $(96.0 \%, 94.4 \%$, and $93.3 \%$ white, respectively, $p=0.03$ ).
Baseline Self-Weighing Frequency and Weight Change

Weight change from baseline to 1-year follow-up was examined as a function of baseline self-weighing. Weight change was $2.2 \pm 5.1 \mathrm{~kg}$ for participants who weighed themselves at least daily, $2.3 \pm 6.0 \mathrm{~kg}$ for participants who weighed themselves less than daily but at least weekly, and $1.7 \pm 5.9 \mathrm{~kg}$ for participants who weighed themselves less than once per week $(p=0.12)$. The difference between groups remained non-significant $(p=0.24)$ when all significant variables listed in Table 2 (i.e., those on which participants differed at baseline) were entered as covariates in the ANOVA.

\section{Change in Self-Weighing Frequency}

Change in self-weighing frequency (calculated from the 3 collapsed categories) from baseline to 1-year follow-up also was examined. During that time period, $72.7 \%$ of participants reported no change in their self-weighing frequency, $9.6 \%$ reported self-weighing more frequently, and $17.7 \%$ reported self-weighing less frequently. As shown in Figure 1 , weight gain at 1-year follow-up was significantly greater $(p<0.001)$ for those participants whose self-weighing frequency had decreased $(4.0 \pm 6.3 \mathrm{~kg})$ compared with those whose frequency had increased $(1.1 \pm 6.5 \mathrm{~kg})$ or remained the same $(1.8 \pm 5.3 \mathrm{~kg})$. When all significant variables listed in Table 2 were entered as covariates, this difference remained significant $(p<0.001$; adjusted means $4.1 \mathrm{~kg}, 1.2 \mathrm{~kg}$, and $2.0 \mathrm{~kg}$, respectively).

Analyses also were conducted to determine if changes in self-weighing frequency were associated with changes in

Table 2. Characteristics of participants by self-weighing frequency

\begin{tabular}{lccc}
\hline \multicolumn{1}{c}{ Characteristic } & $\begin{array}{c}\text { Weighing daily or } \\
\text { more }(\boldsymbol{n}=\mathbf{1 0 8 8})\end{array}$ & $\begin{array}{c}\text { Weighing several times/wk } \\
\text { or weekly }(\boldsymbol{n}=\mathbf{1 2 7 4})\end{array}$ & $\begin{array}{c}\text { Weighing less than } \\
\text { weekly }(\boldsymbol{n}=\mathbf{6 4 1})\end{array}$ \\
\hline Age (yrs) & $50.5 \pm 12.4$ & $47.5 \pm 12.4^{*}$ & $44.6 \pm 12.4^{*} \dagger$ \\
BMI & $24.8 \pm 4.2$ & $25.7 \pm 5.1^{*}$ & $25.1 \pm 5.3 \dagger$ \\
BMI at maximum lifetime weight & $36.3 \pm 7.8$ & $37.7 \pm 8.9^{*}$ & $37.2 \pm 10.0$ \\
Weight maintenance duration (yrs) & $5.5 \pm 7.5$ & $5.4 \pm 7.1$ & $7.1 \pm 8.9^{*} \dagger$ \\
Lifetime intentional weight loss (kg) & $280.0 \pm 258.8$ & $294.0 \pm 263.6$ & $289.0 \pm 244.3$ \\
Cognitive restraint & $15.4 \pm 3.6$ & $15.0 \pm 3.7 *$ & $13.4 \pm 4.2^{*} \dagger$ \\
Disinhibition & $6.9 \pm 3.6$ & $6.8 \pm 3.6$ & $6.4 \pm 3.6^{*}$ \\
Hunger & $4.6 \pm 3.2$ & $4.6 \pm 3.1$ & $4.3 \pm 3.1$ \\
\% calories from fat & $30.4 \pm 0.4$ & $28.5 \pm 0.4 *$ & $29.4 \pm 0.5$ \\
Caloric intake $(\mathrm{kcal} / \mathrm{d})$ & $1407.0 \pm 17.0$ & $1386.1 \pm 15.5$ & $1416.3 \pm 22.3$ \\
Caloric expenditure $(\mathrm{kcal} / \mathrm{wk})$ & $2559.5 \pm 69.9$ & $2479.4 \pm 64.3$ & $2512.6 \pm 91.3$
\end{tabular}

Comparisons for percent of calories from fat, caloric intake, and caloric expenditure were adjusted for age, BMI, and gender. Values presented for these three variables are adjusted mean \pm standard error.

* Significantly differs from daily or more group $(p<0.05)$.

$\dagger$ Significantly differs from several times per week or weekly group $(p<0.05)$. 


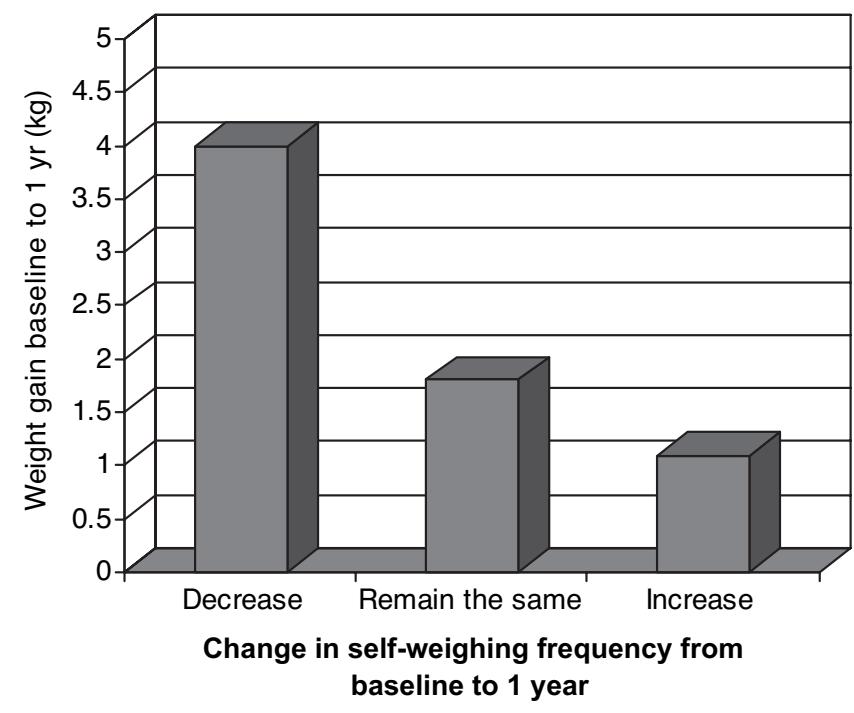

Figure 1: Mean weight gain for participants whose self-weighing frequency decreased, remained the same, or increased from baseline to 1 year.

other weight control variables from baseline to 1-year follow-up. As shown in Table 3, participants whose frequency of self-weighing had decreased were more likely to report increases in their percentage of caloric intake from fat, increases in disinhibition, and decreases in cognitive restraint. There were no differences between groups in change in total caloric intake or physical activity. These results remained the same when gender, BMI, and age were entered into the model.

Because changes in self-weighing frequency were associated with other behavior changes, we reexamined the relationship between change in self-weighing and weight change after adjusting for changes in fat intake, cognitive restraint, and disinhibition. When change scores for these three variables were entered as covariates, the difference in weight change between participants whose weight monitoring frequency had decreased, increased, or remained the same was still significant (adjusted means $3.7 \mathrm{~kg}, 1.2 \mathrm{~kg}$, and $1.9 \mathrm{~kg}$, respectively, $p<0.001)$.

\section{Discussion}

The NWCR has served as an important source of information for identifying a number of behaviors that are associated with successful weight loss maintenance. It has previously been reported that when participants enroll in the NWCR, they typically report engaging in frequent selfmonitoring of their weight. The current, larger study confirmed that finding and showed that $36 \%$ of successful weight losers reported weighing themselves at least once a day, and $79 \%$ weighed themselves at least weekly. Moreover, this study examined additional characteristics of frequent self-weighers. Those individuals who weighed most frequently reported higher levels of disinhibition and higher levels of cognitive restraint; thus, these individuals may be the ones who have the greatest difficulties controlling tendencies to overeat. Although disinhibition scores in this group were higher than in participants who engaged in less frequent self-weighing (6.9 vs. 6.4), their scores were still substantially lower than clinical populations of binge eaters (mean score, 13.3) or bulimia nervosa patients (mean score, 12.4) (17). Similarly, cognitive restraint scores were consistent with those typically reported in individuals undergoing weight loss treatment (mean score, 15.4) (17). In any case, it is possible that frequent self-weighing and other behavioral strategies serve to increase dietary vigilance and thereby help these individuals maintain their successful weight loss. Interestingly, frequent self-weighing was also related to shorter duration of weight loss maintenance before enrollment in the NWCR. Thus, it is possible that participants who have been successful at weight loss maintenance for a longer period of time may be able to regulate

Table 3. Relationship between changes in self-weighing frequency and changes in other weight control variables

\begin{tabular}{lccc}
\hline & $\begin{array}{c}\text { Increased self- } \\
\text { weighing frequency }\end{array}$ & $\begin{array}{c}\text { Decreased self- } \\
\text { weighing frequency }\end{array}$ & Remained the same \\
\hline \% calories from fat & $-0.6 \pm 7.7 \dagger$ & $1.6 \pm 6.7^{*}$ & $0.7 \pm 6.9^{*} \dagger$ \\
Caloric intake (kcal/d) & $-37.1 \pm 444.3$ & $-36.2 \pm 515.0$ & $-14.3 \pm 433.5$ \\
Caloric expenditure (kcal/wk) & $8.7 \pm 1571.5$ & $-169.0 \pm 1940.2$ & $-159.6 \pm 2015.5$ \\
Cognitive restraint & $0.2 \pm 2.9 \dagger$ & $-1.1 \pm 3.2$ & $-0.2 \pm 2.7 \dagger$ \\
Disinhibition & $0.2 \pm 2.4$ & $0.6 \pm 2.6$ & $0.1 \pm 2.4 \dagger$
\end{tabular}

Change scores are calculated as values at 1 year follow-up minus values at baseline.

* Significantly differs from increased frequency group $(p<0.05)$.

$\dagger$ Significantly differs from decreased frequency group $(p<0.05)$. 
their weight and weight control behaviors without frequently monitoring their weight.

A significant finding from this study was that maintaining or increasing self-weighing frequency from baseline to 1-year follow-up was associated with less weight regain. Consistent and more intensive self-weighing may allow individuals to catch weight gains before they escalate and make behavior changes to prevent additional weight gain. In previous research, we found that recovery from weight regain was rare but possible if individuals caught the weight gains early, reversing small (as opposed to larger) weight gains (18). Consistent self-weighing may facilitate weight control by enabling individuals to catch and reverse small weight gains. Alternatively, it is possible that individuals who are gaining weight decrease their frequency of selfweighing because they find doing so distressing or because they do not perceive it as a helpful weight control behavior.

The findings on the importance of frequent self-weighing for maintenance of weight loss are similar to results reported recently for the STOP Regain trial (7). In this randomized clinical trial, 314 successful weight losers were randomly assigned to a control group or to a self-regulation intervention via the Internet or face-to-face. The self-regulation intervention taught participants to weigh themselves daily and to use the information from the scale to identify small weight gains and to immediately implement appropriate changes in eating and exercise behavior. The interventions were effective in decreasing the proportion of participants who regained $\geq 5$ lbs over the 18 -month trial; moreover, within the intervention groups, those individuals who weighed themselves daily were $82 \%$ more likely to maintain their weight loss compared with those who weighed less often.

In the current study, self-weighing appeared to go hand in hand with other weight control behaviors. Participants who decreased their self-weighing frequency reported increases in their percentage of caloric intake from fat, increases in disinhibition, and decreases in cognitive restraint from baseline to 1-year follow-up. Interestingly, when changes in fat intake, disinhibition, and cognitive restraint were accounted for, maintaining or increasing self-weighing frequency from baseline to 1-year follow-up continued to be associated with significantly less weight gain than decreasing self-weighing frequency. This suggests that, while change in self-weighing frequency is, to some extent, a marker for changes in other parameters of weight control, decreasing self-weighing frequency is also independently associated with greater weight regain.

There are several limitations to this study. The crosssectional nature of these findings precludes drawing conclusions about whether consistent self-weighing has a causal influence on weight loss maintenance. Future observational studies that include more frequent assessments would be useful in determining whether weight change or change in self-weighing frequency occurs first. Because the demographic composition of this sample was relatively homogenous, these findings may not generalize to ethnic minorities, to those with lower socioeconomic status, or to those who are trying to achieve weight loss, as opposed to maintain their weight loss. This study also is limited by the self-report of body weight and self-weighing frequency and the lack of validation of the assessment of self-weighing.

In summary, this study suggests that frequent self-weighing may help with long-term maintenance of weight loss. Linde et al. (6) have suggested that frequent self-weighing may not only be important for weight loss maintenance but may also be associated with better outcomes in a weight loss intervention and in the prevention of weight gain. Experimental studies on weight monitoring frequency are needed to determine if frequent self-weighing can improve initial weight loss as well as help prevent regain.

\section{Acknowledgment}

This research was supported by NIH Grant DK066787 (to R.R.W.).

\section{References}

1. Klem ML, Wing RR, McGuire MT, Seagle HM, Hill JO. A descriptive study of individuals successful at long-term maintenance of substantial weight loss. Am J Clin Nutr. 1997;66: $239-46$.

2. Wyatt HR, Grunwald GK, Mosca CL, Klem ML, Wing RR, Hill JO. Long-term weight loss and breakfast in subjects in the National Weight Control Registry. Obes Res. 2002;10:78-82.

3. Raynor DA, Phelan S, Hill JO, Wing RR. Television viewing and long-term weight maintenance: results from the National Weight Control Registry. Obesity. 2006;14:1816-24.

4. Kanfer FH, Karoly P. Self-control: a behavioristic excursion into the lion's den. Behav Ther. 1972;3:398-416.

5. Kirschenbaum DS. Self-regulatory failure: a review with clinical implications. Clin Psychol Rev. 1987;7:77-104.

6. Linde JA, Jeffery RW, French SA, Pronk NP, Boyle RG. Self-weighing in weight gain prevention and weight loss trials. Ann Behav Med. 2005;30:210-6.

7. Wing RR, Tate DF, Gorin AA, Raynor HA, Fava JL. A self-regulation program for maintenance of weight loss. N Engl J Med. 2006;355:1563-71.

8. McGuire MT, Wing RR, Klem ML, Lang W, Hill JO. What predicts weight regain in a group of successful weight losers? J Consult Clin Psychol. 1999;67:177-85.

9. Stunkard AJ, Messick S. The three-factor eating questionnaire to measure dietary restraint, disinhibition and hunger. J Psychosom Res. 1985;29:71-83.

10. Allison DB, Kalinsky LB, Gorman BS. A comparison of the psychometric properties of three measures of dietary restraint. Psychol Assess. 1992;4:391-398.

11. Paffenbarger RS, Wing AL, Hyde RT. Physical activity was an index of heart attack risk in college alumni. Am J Epidemiol. 1978;108:161-75. 
12. Washburn RA, Smith KW, Goldfield SRW, McKinlay JB. Reliability and physiologic correlates of the Harvard Alumni Activity Survey in the general population. J Clin Epidemiol. 1991;44:1319-26.

13. Siconolfi SF, Lasater TM, Snow RCK, Carleton RA. Selfreported physical activity compared with maximal oxygen uptake. Am J Epidemiol. 1985;122:101-5.

14. Boucher B, Cotterchio M, Kreiger N, Nadalin V, Block T, Block G. Validity and reliability of the Block98 foodfrequency questionnaire in a sample of Canadian women. Public Health Nutr. 2006;9:84-93.

15. Block G, Hartman AM, Dresser CM, Carroll MD, Gannon
J, Gardner I. A data-based approach to diet questionnaire testing and design. Am J Epidemiol. 1986;124:45369.

16. Block G, Woods M, Potosky A, Clifford C. Validation of a self-administered diet history questionnaire using multiple diet records. J Clin Epidemiol. 1990;43:1327-35.

17. Klem ML, Wing RR, McGuire MT, Seagle HM, Hill JO. Psychological symptoms in individuals successful at longterm weight loss. Health Psychol. 1998;17:336-45.

18. Phelan S, Hill JO, Lang W, Dibello JR, Wing RR. Recovery from relapse among successful weight maintainers. Am J Clin Nutr. 2003;78:1079-84. 